\title{
Variación espacio-temporal de la estructura ecológica de la comunidad de peces en la Laguna del Ostión, Veracruz, México
}

\section{Spatio-temporal variation on the ecological structure of the fish community in the Ostión Lagoon, Veracruz, Mexico}

\author{
Arturo Aguirre-León ${ }^{1 *}$, Silvia Díaz-Ruiz² y Mariela Gazca-Castro ${ }^{1}$
}

\begin{abstract}
RESUMEN
La comunidad de peces en la Laguna del Ostión es objeto de pesca, sin embargo, los estudios ecológicos sobre esta son escasos. Se analizó la variación espacial y temporal de su estructura comunitaria, así como de los parámetros fisicoquímicos de salinidad, temperatura, oxígeno disuelto, profundidad y transparencia de la laguna. Los peces fueron recolectados bimensualmente de marzo de 2007 a enero de 2008, en ocho estaciones de muestreo con una red de arrastre camaronera. Se evaluaron diferencias significativas espacio-temporales de diversidad, riqueza, equitatividad, densidad, biomasa y peso promedio, mediante análisis de varianza. Se realizó análisis de correspondencias canónicas (ACC) para determinar las asociaciones de peces y su correlación con los factores ambientales del sistema. Se recolectaron 737 individuos con peso total de $19081.90 \mathrm{~g}$. Se identificaron 19 familias, 23 géneros y 34 especies, 12 de las cuales fueron nuevos registros para la laguna. Cuatro especies fueron dominantes en el sistema. Los parámetros comunitarios mostraron relación con los gradientes ambientales y las temporadas climáticas. La diversidad fue más alta en mayo $\left(\mathrm{H}^{\prime}=2.36\right)$ y enero $\left(\mathrm{H}^{\prime}=2.48\right)$ y la densidad en julio $\left(0.018\right.$ ind. $\left./ \mathrm{m}^{2}\right)$. El ACC mostró que la composición y distribución espacio-temporal de la comunidad de peces estuvo condicionada por la salinidad, la temperatura, el oxígeno disuelto y la profundidad. El conocimiento de la estructura comunitaria, las funciones biológicas de los peces y su interacción con los factores ambientales de esta laguna es relevante, ya que no se han desarrollado estudios continuos en este sistema.
\end{abstract}

Palabras clave: diversidad, abundancia, dominancia, categorías tróficas, asociaciones de peces.

1 Departamento El Hombre y su Ambiente. Laboratorio Ecología Costera y Pesquerías. Universidad Autónoma Metropolitana-Xochimilco. El Hueso 1100, Col. Villa Quietud, Ciudad de México 04960, México; agla1269@correo.xoc.uam.mx* ORCID: https://orcid.org/0000-0002-2882-3258, mgc14bio@gmail.com ORCID: https://orcid. org/0000-0002-5036-2902

2 Departamento de Hidrobiología. Laboratorio Ictiología y Ecología Costera. Universidad Autónoma Metropolitana-Iztapalapa. San Rafael Atlixco 186, Col. Vicentina, Ciudad de México 09340, México; sdr@xanum.uam.mx ORCID: https://orcid.org/0000-0003-3035-9017 


\begin{abstract}
Although the fish community in the Ostión Lagoon is subject to fishing, ecological studies on it are scarce. The spatial and temporal variation of this community structure was analyzed, as well as the physicochemical parameters of the lagoon's salinity, temperature, dissolved oxygen, depth, and transparency. Samples were collected bimonthly from March 2007 to January 2008 in eight sampling stations with a shrimp trawl. Significant spatial and temporal differences were evaluated in diversity, richness, evenness, density, biomass, and average weight through analysis of variance. A canonical correspondence analysis (CCA) was used to determine fish associations and their correlation with the environmental factors of the system. A total of 737 individuals were collected with a total weight of 19,081.90 g. The study identified 19 families, 23 genera, and 34 species, of which 12 were new records for the lagoon. Four species were found to be dominant in the system. Community parameters showed a relationship with environmental gradients and climatic seasons. Diversity was higher in May $\left(\mathrm{H}^{\prime}=2.36\right)$ and January $\left(\mathrm{H}^{\prime}=2.48\right)$, while density was higher in July $\left(0.018\right.$ ind $\left./ \mathrm{m}^{2}\right)$. The CCA showed that the composition and spatio-temporal distribution of the fish community was conditioned by salinity, temperature, dissolved oxygen, and depth. Knowing the fish's community structure and biological functions and their interaction with environmental factors in this lagoon is relevant since ongoing studies have not been conducted for this system.
\end{abstract}

Keywords: diversity, abundance, dominance, trophic categories, fish associations.

\section{INTRODUCCIÓN}

El Estado de Veracruz ocupa el tercer lugar de importancia por su extensión lagunar-estuarina en el golfo de México, con alta biodiversidad y gran variedad de recursos pesqueros (Lara-Domínguez et al. 2011). Estos sistemas costeros poseen características propias geomorfológicas, hidrológicas y cuantiosa diversidad de hábitats, lo que condiciona los patrones de utilización de estos por poblaciones de peces y se refleja en la composición, diversidad, distribución y abundancia de las especies en espacio y tiempo (Cowan et al. 2013; Sánchez-Gil et al. 2019). El estudio de este grupo faunístico tiene un gran interés ecológico y económico, ya que, un gran número de especies sujetas a explotación comercial dependen de estos ecosistemas litorales para completar alguna parte de sus ciclos biológicos (França et al. 2012; Abrantes et al. 2015; Chi-Espínola \& Vega-Cendejas, 2016; Aguirre-León et al. 2018).

La Laguna del Ostión, la más sureña del estado de Veracruz, presenta una pesca artesanal histórica dirigida, principalmente, al ostión y camarón (Lara-Domínguez et al. 2009). La pesca de escama costera para consumo local incluye las especies Eugerres plumieri, Lutjanus campechanus, Mugil curema, Caranx hippos, Lutjanus griseus, Scomberomorus cavalla, Centropomus parallelus, Orthopristis chrysoptera, Ocyurus chrysurus, entre otros (Díaz-Ruiz et al. 2008). 
Esta laguna, como otras del golfo de México, ha sido alterada por procesos climáticos, que afectan la temporalidad regional por el incremento de huracanes de gran intensidad, la erosión de playas y el cambio del nivel del mar (Lango-Reynoso et al. 2019). Al mismo tiempo, las actividades humanas industriales han inducido, por ejemplo, contaminación por petróleo y la sobrepesca, por su parte, ha perturbado la integridad del ecosistema por varias décadas (Solano \& Frutos, 2013; Beltrán-García et al. 2019). Esto ha modificado la organización de los sistemas y sus comunidades ícticas, llevando a la pérdida de biodiversidad y la consecuente baja en la disponibilidad de recursos pesqueros (Baltz \& Yáñez-Arancibia, 2013; Sánchez-Gil et al. 2019). La Laguna del Ostión está ubicada en un área costera con desarrollo industrial y pesquero donde debe seguirse realizando investigación ecológica sobre la estructura y función de la comunidad de peces, debido a la escasa información sobre el estado actual del sistema (Díaz-Ruiz et al. 2008). El trabajo de Bozada \& Chávez (1986) es el estudio ambiental y de composición de peces más conocido para esta laguna, a partir del cual han transcurrido décadas sin investigaciones publicadas hasta la fecha. A partir de dicho estudio, con datos del 2007 al 2008, se cubren faltantes de información que permiten establecer un marco de referencia para comparaciones futuras, sobre posibles cambios en la estructura comunitaria de peces de este sistema. Por lo anterior, se plantearon los siguientes objetivos: 1) determinar diversidad, riqueza, equitatividad, densidad, biomasa y peso promedio de la comunidad íctica de la laguna; 2) definir especies dominantes, componentes ícticos y categorías tróficas; 3) caracterizar las diferentes asociaciones de peces y su relación con los factores fisicoquímicos que condicionan su composición y distribución.

\section{MATERIALES Y MÉTODOS}

Área de estudio: La Laguna del Ostión se localiza en el golfo de México, al sur de la planicie costera de Veracruz $\left(18^{\circ} 09^{\prime}-18^{\circ} 13^{\prime} \mathrm{N}\right.$ y $94^{\circ} 35^{\prime}$ $94^{\circ} 37^{\prime} \mathrm{W}$ ) (Fig. 1), cercana a la ciudad industrial de Coatzacoalcos. Tiene una superficie de $12.7 \mathrm{~km}^{2}$, con una profundidad de 0.5 a $2.64 \mathrm{~m}$. Presenta una barrera arenosa y posee comunicación permanente con el mar, a través de la boca Jicacal ubicada al noreste del sistema, lo cual condiciona el flujo y reflujo de agua salina, así como el intercambio de nutrientes y fauna marina (Fig. 1). Recibe importantes aportes de agua dulce de los ríos Metzapa, Huazuntlán y Temoloapan, además de otros escurrimientos de menor importancia. El clima es cálido húmedo Am (f) con lluvias en verano; la temperatura media anual fluctúa entre $18^{\circ} \mathrm{C}$ y 


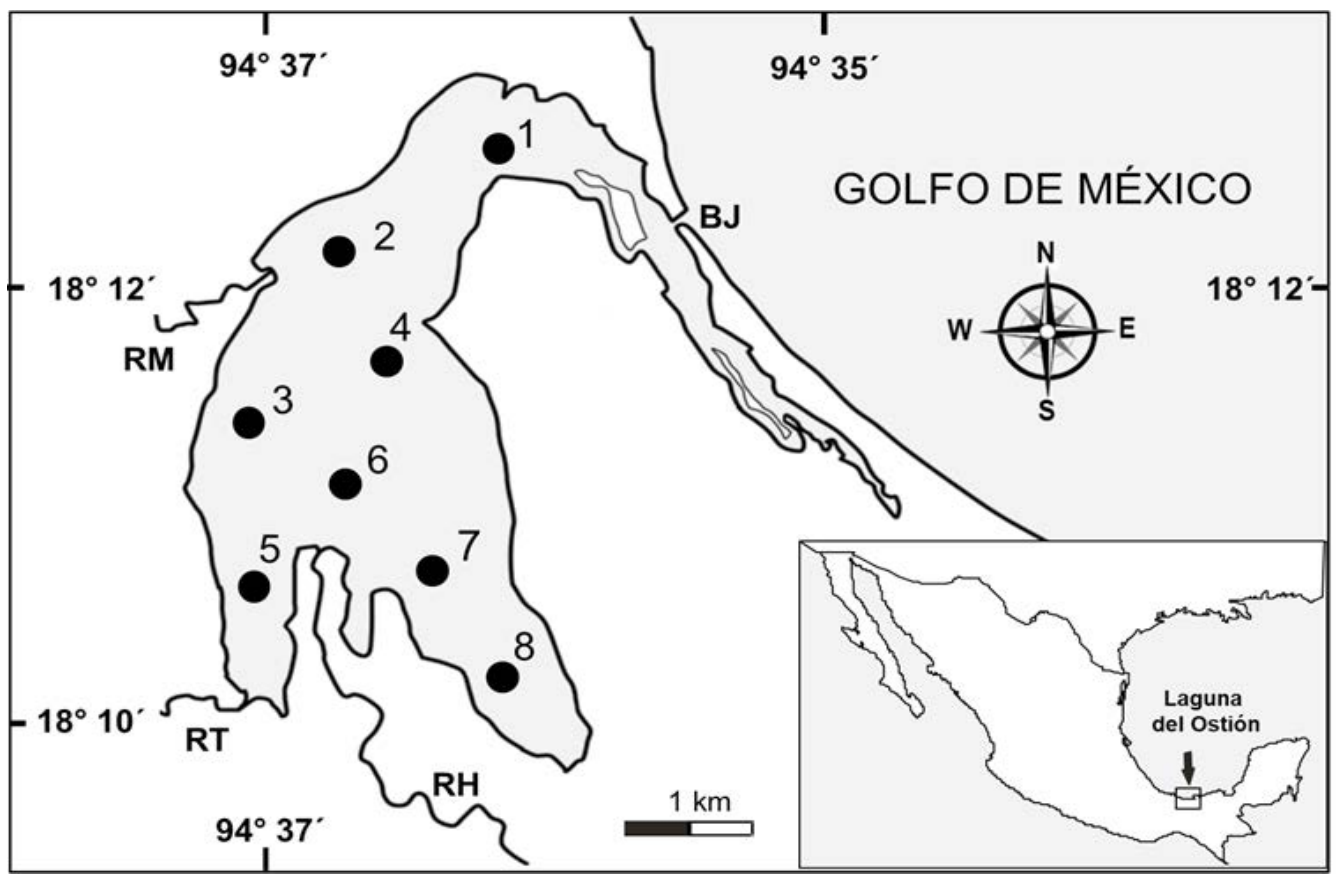

Fig. 1. Sistema lagunar del Ostión, Veracruz. Se indica la ubicación de las estaciones de muestreo $(\bullet)$, las principales características fisiográficas del área de estudio y los ríos que drenan en el sistema. Río Metzapa (RM), río Temoloapan (RT), río Huazuntlán (RH) y boca marina Jicacal (BJ)

Fig. 1. Ostión Lagoon System, Veracruz. The location of sampling stations (•), the main physiographic features of the study area, and the rivers draining into the system are shown. Metzapa River (RM), Temoloapan River (RT), Huazuntlán River (RH), and Jicacal marine inlet (BJ)

$22^{\circ} \mathrm{C}$ y la precipitación anual es de 1 $376 \mathrm{~mm}$, con lluvia invernal mayor al 10.2\% del total anual (García, 2004). A lo largo del año, se presentan tres temporadas climáticas: secas (febrero-mayo), lluvias (junio-septiembre) y nortes (octubre-febrero) (Bozada \& Chávez, 1986; Lara-Domínguez et al. 2009). Existen diferentes ambientes acuáticos en el sistema, como pantanos de manglar salobre y de baja salinidad (Rhizophora mangle, Laguncularia racemosa, Avicennia germinans), vegetación emergente (Thalia geniculata, Cyperus gigantus, Nymphaea ampla) y sumergida (Ruppia maritima, Syringodium filiforme), al igual que áreas de alta sedimentación (Díaz-Ruiz et al. 2008). 
Actividades de campo: La recolección de peces se realizó en seis campañas entre marzo de 2007 y enero de 2008, cada dos meses, abarcando las épocas climáticas del año, en ocho estaciones de muestreo por evento, para un total de 48 lances. Las capturas fueron diurnas (09:00 - 15:00 horas), con una red de arrastre camaronera de $5.0 \mathrm{~m}$ de largo, $2.5 \mathrm{~m}$ de abertura de trabajo y luz de malla de $1.9 \mathrm{~cm}$, en una lancha de $7 \mathrm{~m}$ de eslora y motor fuera de borda de $50 \mathrm{HP}$. Cada lance tuvo una duración de 10 minutos y se cubrió un área de arrastre aproximada de $1500 \mathrm{~m}^{2}$ por estación. La estimación del área barrida se hizo siguiendo lo descrito por Sparre y Venema (1997). Todos los peces capturados fueron fijados en formaldehído al $4 \%$ neutralizado con borato de sodio y, posteriormente, preservados en etanol al 70\% para su análisis en el laboratorio. En cada mes, se registró, por estación a media agua, salinidad (UPS), temperatura $\left({ }^{\circ} \mathrm{C}\right)$ y oxígeno disuelto $(\mathrm{mg} / \mathrm{L})$, con un multianalizador YSI-3800; la profundidad, con una sonda graduada (m), y la transparencia del agua, con un disco de Secchi (m).

Actividades de laboratorio: Se midió la longitud total (LT) de todos los peces capturados, con un ictiómetro graduado $(\mathrm{cm})$, y su peso individual, con una balanza digital SartoriusEntris 2202-1S (precisión $0.01 \mathrm{~g}$ ). La identificación taxonómica de los peces se realizó siguiendo las claves del
Atlántico occidental y del Golfo de México (Fischer, 1995; Castro-Aguirre et al. 1999; Miller et al. 2009). El estatus taxonómico de cada grupo supraespecífico se basó en el criterio de Nelson (2016) y, para corroborar la autoridad y nomenclatura actual de las especies, se consultó Fricke et al. (2020).

Se calcularon los índices ecológicos de la comunidad: diversidad H' (Shannon \& Weaver, 1963), riqueza de especies D (Margalef, 1995) y equitatividad $\mathrm{J}^{\prime}$ (Pielou, 1966), así como los parámetros, densidad (ind./ $\left.\mathrm{m}^{2}\right)$, biomasa $\left(\mathrm{g} / \mathrm{m}^{2}\right)$ y peso promedio de los peces (g/ind.) (Aguirre-León et al. 2014). La dominancia de las especies se determinó mediante el índice de valor de importancia (IVI), utilizando el programa ANACOM (De la Cruz-Agüero, 1994). Se establecieron los diferentes componentes ícticos de la laguna, de acuerdo con el criterio de Castro-Aguirre et al. (1999), los cuales se clasifican como: $\mathrm{E}$ = estuarino, $\mathrm{EM}$ = eurihalino marino, $\mathrm{ESM}=$ estenohalino marino y $\mathrm{D}=$ dulceacuícola. Los peces se agruparon en categorías tróficas, según su alimentación y siguiendo los criterios propuestos por Froese \& Pauly (2019) y Robertson et al. (2019) en planctívoros (PL), especies que se alimentan predominantemente de plancton; omnívoros (OM), especies que se alimentan de invertebrados bentónicos y vegetales; detritívoros (DE), especies que se alimentan principalmente de detritus, invertebrados 
bentónicos y vegetales; carnívoros (CA), peces que se alimentan de invertebrados bentónicos o peces.

Análisis estadístico: Se aplicó análisis de varianza, con la finalidad de indicar diferencias estadísticas en espacio (entre estaciones) y por tiempo (entre meses) tanto de las variables fisicoquímicas como de los indicadores ecológicos comunitarios. Previamente, fue evaluado el supuesto de homogeneidad de varianzas entre grupos de datos, empleando la prueba de Levene (1960). Cuando fue necesario, se aplicaron transformaciones logarítmicas. Las variables con varianzas homogéneas se trataron con ANOVA-F- y las heterogéneas con Kruskal-Wallis-H- y nivel de significancia $P<0.05$ (Zar, 2010; Heiman, 2011). Los análisis se realizaron mediante el programa estadístico STATISTICA (Stat Soft, 2013).

Se usaron datos históricos de precipitación (CNA-SMN, 2020) para indicar la variación mensual entre temporadas climáticas.

Para validar el esfuerzo del muestreo, se realizaron curvas de acumulación de especies, por medio de los estimadores no-paramétricos Chao2, Jacknife1, Jacknife2 y Bootstrap. Se construyó una base de datos de presencia/ausencia de especies para cada estación y mes de muestreo (Magurran et al. 2011). Las curvas de acumulación se construyeron con 9999 aleatorizaciones, a través del programa estadístico PRIMER-E (Clarke y Warwick, 2006).

Para definir las asociaciones de peces presentes en el sistema, se dispuso un análisis de correspondencias canónicas (ACC) (Ter Braak, \& Verdonschot, 1995), para lo cual se elaboraron dos matrices, una de abundancia de las especies por estación y mes, y otra de variables fisicoquímicas. Fueron utilizadas las correlaciones inter-set para establecer qué parámetros ambientales condicionaron distribución, abundancia y composición de los conjuntos de peces (McGarigal et al. 2000). El ACC fue generado con el programa estadístico MVSP (KCS, Inc, 2013).

\section{RESULTADOS}

El análisis espacial de los factores fisicoquímicos del sistema (Cuadro 1) mostró, en general, valores mayores en la parte norte (estaciones 1 y 2), por la cercanía de la boca marina, y menores en la porción sur (estaciones de la 5 a la 8) por la influencia fluvial (Fig. 1). La única variable que indicó diferencias significativas entre sitios de muestreo fue la profundidad (ANOVA-F $(7,40)=14.89, P<0.0001)$. En escala temporal, la salinidad y la transparencia del agua fueron mayores en secas, la temperatura en lluvias y el oxígeno disuelto y la profundidad en nortes (Cuadro 1). Se encontraron diferencias 


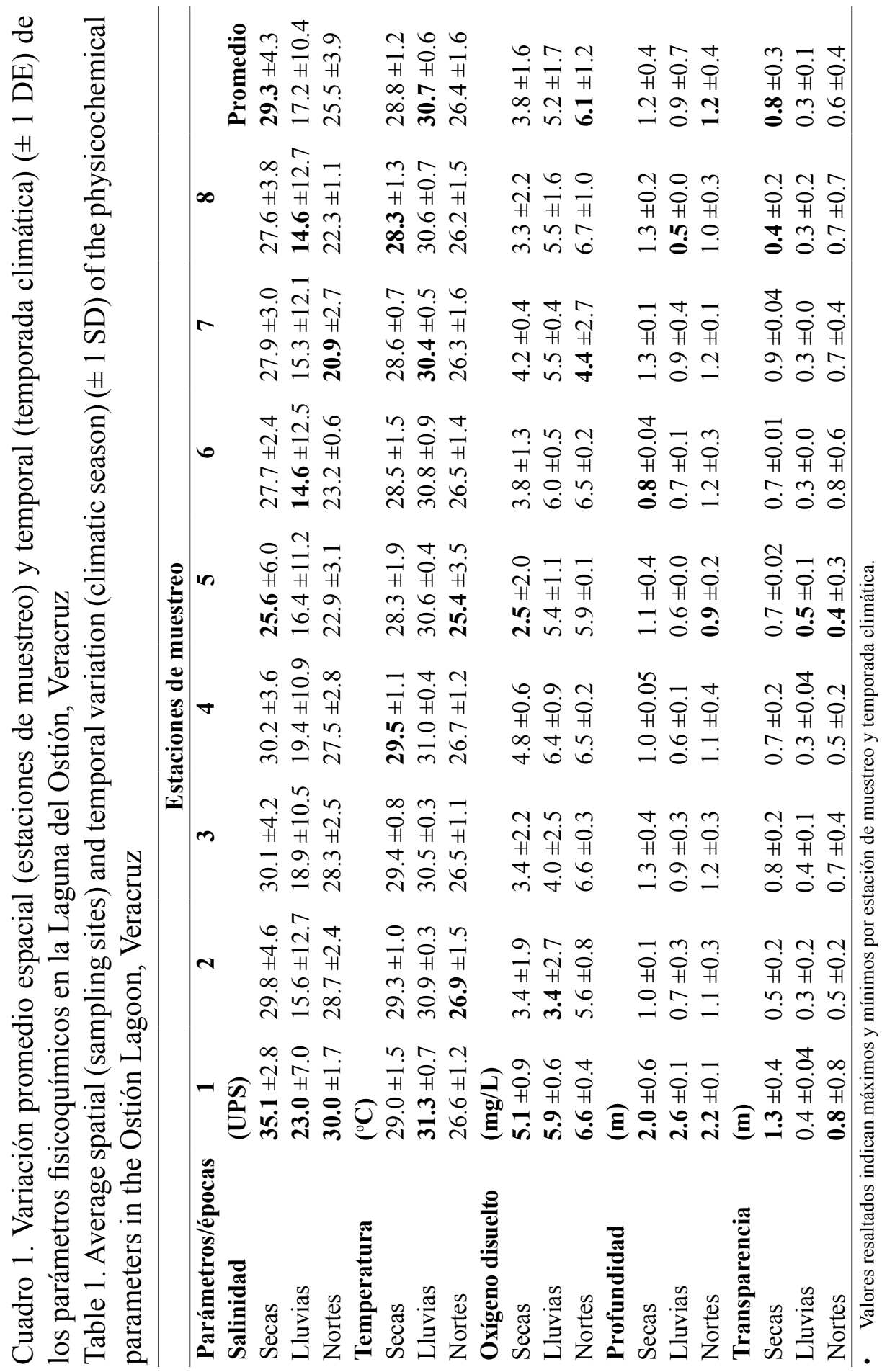


Cuadro 2. Listado de las especies de peces colectadas en el sistema lagunar del Ostión, Veracruz

Table 2. List of the fish species collected in the Ostión Lagoon System, Veracruz

\begin{tabular}{|c|c|c|c|c|c|c|c|c|}
\hline Familia & Especies & $\mathbf{A B}$ & $\begin{array}{c}\text { Número } \\
(\%)\end{array}$ & $\begin{array}{r}\text { Peso } \\
\text { (\%) }\end{array}$ & $\begin{array}{c}\text { Frecuencia } \\
(\%)\end{array}$ & $\begin{array}{l}\text { IVI } \\
(\%)\end{array}$ & $\mathrm{CC}$ & CT \\
\hline Gymnuridae & Gymnura micrura & Gmi & 0.14 & 2.63 & 0.45 & 3.22 & ESM & $\mathrm{CA}$ \\
\hline Dasyatidae & Hypanus sabinus & Hsa & 1.63 & 6.86 & 2.27 & 10.76 & E & $\mathrm{CA}$ \\
\hline Dasyatidae & Hypanus americana & Ham & 0.54 & 7.36 & 1.36 & 9.27 & ESM & $\mathrm{CA}$ \\
\hline Engraulidae & Anchovia clupeoides & Acl & 1.36 & 0.40 & 0.91 & 2.66 & EM & PL \\
\hline Engraulidae & Anchoa mitchilli & Ami & 0.54 & 0.03 & 0.91 & 1.48 & EM & PL \\
\hline Ariidae & Cathorops aguadulce & Cag & 8.96 & 10.86 & 7.73 & 27.54 & E & CA \\
\hline Synodontidae & Synodus foetens & Sfo & 0.27 & 0.33 & 0.45 & 1.06 & EM & $\mathrm{CA}$ \\
\hline Batrachoidae & Opsanus beta & Obe & 4.88 & 25.56 & 7.73 & 38.18 & EM & $\mathrm{CA}$ \\
\hline Mugilidae & Mugil curema & Mcu & 0.81 & 1.25 & 1.82 & 3.88 & EM & $\mathrm{DE}$ \\
\hline Triglidae & Prionotus punctatus & Ppu & 0.27 & 0.04 & 0.45 & 0.76 & ESM & $\mathrm{CA}$ \\
\hline Centropomidae & Centropomus undecimalis & Cun & 1.22 & 2.61 & 5.00 & 8.83 & EM & $\mathrm{CA}$ \\
\hline Centropomidae & Centropomus pectinatus & Cpe & 1.90 & 2.16 & 4.09 & 8.16 & EM & $\mathrm{CA}$ \\
\hline Centropomidae & Centropomus parallelus & Cpa & 6.65 & 5.78 & 5.45 & 17.89 & EM & $\mathrm{CA}$ \\
\hline Lutjanidae & Lutjanus cyanopterus & Lcy & 0.54 & 0.96 & 0.91 & 2.41 & EM & CA \\
\hline Lutjanidae & Lutjanus griseus & Lgr & 0.27 & 1.72 & 0.91 & 2.90 & EM & $\mathrm{CA}$ \\
\hline Lutjanidae & Lutjanus apodus & Lap & 0.41 & 0.60 & 0.45 & 1.46 & EM & $\mathrm{CA}$ \\
\hline Lutjanidae & Lutjanus analis & Lan & 0.14 & 0.08 & 0.45 & 0.67 & EM & CA \\
\hline Gerreidae & Eucinostomus melanopterus & Eme & 7.33 & 1.38 & 5.91 & 14.61 & EM & $\mathrm{CA}$ \\
\hline Gerreidae & Eucinostomus gula & Egu & 5.29 & 1.08 & 2.27 & 8.64 & EM & CA \\
\hline Gerreidae & Eucinostomus argenteus & Ear & 1.09 & 0.17 & 2.73 & 3.99 & EM & $\mathrm{CA}$ \\
\hline Gerreidae & Diapterus rhombeus & Drh & 17.91 & 3.28 & 8.64 & 29.83 & EM & $\mathrm{OM}$ \\
\hline Gerreidae & Diapterus auratus & Dau & 4.88 & 1.14 & 2.73 & 8.75 & EM & $\mathrm{OM}$ \\
\hline Gerreidae & Eugerres plumieri & Epl & 2.17 & 2.79 & 3.18 & 8.14 & EM & $\mathrm{CA}$ \\
\hline Sparidae & Archosargus probatocephalus & Apr & 3.66 & 9.24 & 5.91 & 18.81 & EM & $\mathrm{OM}$ \\
\hline Scianidae & Bairdiella chrysoura & Bch & 0.27 & 0.34 & 0.91 & 1.52 & EM & $\mathrm{CA}$ \\
\hline Scianidae & Bairdiella rochus & Bro & 1.22 & 3.23 & 2.73 & 7.18 & EM & $\mathrm{CA}$ \\
\hline Cichlidae & Oreochromis niloticus & Oni & 0.14 & 1.31 & 0.45 & 1.90 & $\mathrm{D}$ & $\mathrm{DE}$ \\
\hline Eleotridae & Gobiomorus dormitor & Gdo & 0.14 & 0.70 & 0.45 & 1.29 & $\mathrm{E}$ & $\mathrm{CA}$ \\
\hline Gobiidae & Bathygobius soporator & Bso & 0.14 & 0.01 & 0.45 & 0.60 & EM & CA \\
\hline Gobiidae & Gobionellus oceanicus & Goc & 1.76 & 0.49 & 3.18 & 5.43 & ESM & $\mathrm{OM}$ \\
\hline Paralichthyidae & Citharichthys spilopterus & Csp & 20.08 & 5.30 & 13.64 & $39.01 \downarrow$ & EM & CA \\
\hline Paralichthyidae & Citharychthys uhleri & Cuh & 1.22 & 0.15 & 1.36 & 2.73 & EM & CA \\
\hline Achiridae & Achirus lineatus & Ali & 2.04 & 0.14 & 3.64 & 5.81 & EM & $\mathrm{CA}$ \\
\hline Monacanthidae & Stephanolepis hispidus & Shi & 0.14 & 0.03 & 0.45 & 0.62 & ESM & $\mathrm{OM}$ \\
\hline TOTALES & & & 100.0 & 100.0 & 100.0 & 300.0 & & \\
\hline
\end{tabular}

Indice de valor de importancia (IVI): especies dominantes. Componente comunitario (CC): EM = eurihalino marino, $\mathrm{ESM}=$ estenohalino marino, $\mathrm{E}=$ estuarino, $\mathrm{D}=$ dulceacuícola. Categoría trófica $(\mathrm{CT}): \mathrm{PL}=$ planctívoros, $\mathrm{OM}=\mathrm{om}-$ nívoros, $\mathrm{CA}$ = carnívoros, $\mathrm{DE}=$ detritívoros. Abreviaturas $(\mathrm{AB})$ utilizadas en la Figura 5 (análisis de correspondencias canónicas).

Importance Value Index (IVI): $\downarrow$ dominant species. Community inhabitant (CC): EM = marine euryhaline, $\mathrm{ESM}=$ marine stenohaline, $\mathrm{E}=$ estuarine, $\mathrm{D}=$ freshwater. Trophic category $(\mathrm{CT})$ : $\mathrm{PL}=$ planktivore, $\mathrm{OM}=$ omnivore, $\mathrm{CA}=$ carnivore, $\mathrm{DE}=$ detritivore. Abbreviations $(\mathrm{AB})$ used in Figure 5 are shown (Canonical Correspondence Analysis). 
significativas entre temporadas para la salinidad (Kruskal-Wallis-H $(2,48)=$ 14.7355, $P=0.0006)$, la temperatura $($ Kruskal-Wallis-H $(2,48)=37.3802, P$ $<0.0001$ ), el oxígeno disuelto (ANOVA-F $(2,45)=48.3286, P<0.0001)$ y la transparencia (Kruskal-Wallis-H $(2,48)=13.6466, P=0.0011)$.

En la Laguna del Ostión se recolectaron 737 peces, pertenecientes a 12 órdenes, 19 familias, 23 géneros y 34 especies en 48 lances de muestreo (Cuadro 2). Las curvas de acumulación de especies mostraron que el esfuerzo de muestreo representó adecuadamente el número de especies en cada sitio y mes de colecta. El resultado de los estimadores sugiere que el número de especies colectadas fue cercano al valor promedio estimado con 39 especies. La representatividad del muestreo osciló entre el $77 \%$ y el $86 \%$ de las especies esperadas. El valor máximo de número de especies (41) se obtuvo con el estimador Jacknife1 y Jacknife2, mientras que el menor número fue estimado por Chao2 y Boostrap con 38 especies (Fig. 2). Las familias mejor representadas en número de especies

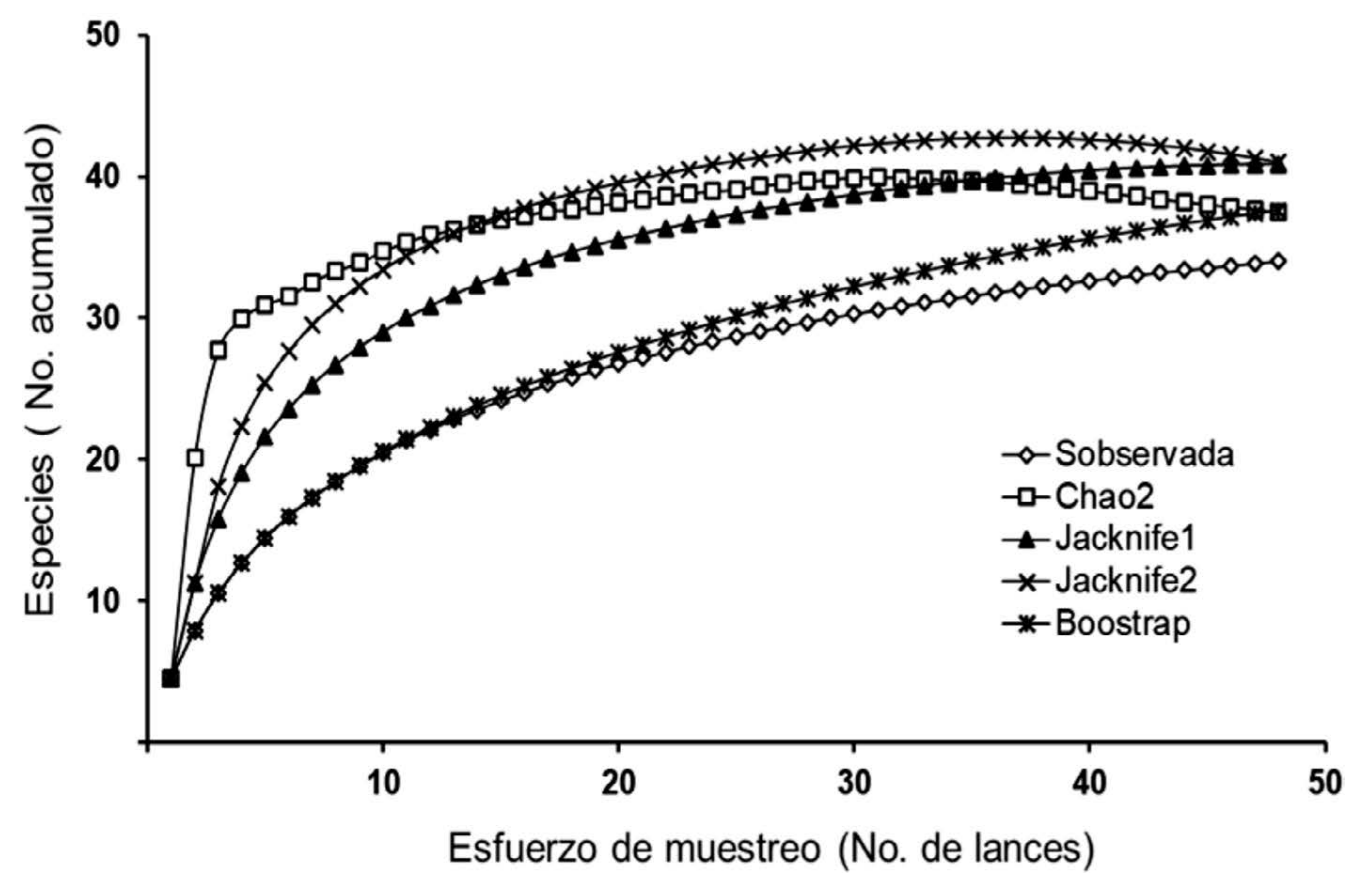

Fig. 2. Curvas estimadas de acumulación de especies de peces capturados en el sistema lagunar del Ostión. Se indican las tendencias de las diferentes pruebas estadísticas calculadas. Fig. 2. Estimated accumulation curves of fish species caught in the Ostión Lagoon System. The trends of the different statistical tests calculated are indicated. 
fueron Gerreidae con seis (Diapterus auratus, D. rhombeus, Eucinostomus argenteus, E. gula, E. melanopterus y Eugerres plumieri), Lutjanidae con cuatro (Lutjanus analis, L. apodus, L. cyanopterus y L. griseus) y Centropomidae con tres (Centropomus parallelus, C. pectinatus y C. undecimalis). El número de especies varió entre $21(62 \%)$, en la estación 1 , y $13(38 \%)$, en la estación 3; y entre $20(59 \%)$, en enero (nortes), y 11 (32\%), en marzo (secas). Del total de especies registradas, ocho $(23.5 \%)$ presentaron distribuciones restringidas en el sistema como Bathygobius soporator, Citharichthys uhleri, Gymnura micrura, L. analis, L. apodus, Prionotus punctatus, Stephanolepis hispidus y Synodus foetens que fueron capturadas únicamente en las estaciones 1 y 2 . Doce especies son registros nuevos para el sistema lagunar del Ostión: G. micrura, Anchovia clupeoides, Mugil curema, C. pectinatus, P. punctatus, L. analis, L. cyanopterus, E. argenteus, Oreochromis niloticus, Gobiomorus dormitor, C. uhleri y S. hispidus.

El análisis espacial de los índices H', D y J' mostró una tendencia similar entre ellos y los relacionados con el gradiente ambiental, así los valores más altos se observaron hacia la boca marina y los más bajos en la zona de mayor influencia fluvial (Fig. 3). El promedio más alto de H' (1.41 $\pm 0.26)$ se registró en la estación 2 y el más bajo (1.02 \pm 0.84$)$ en la 3 (Fig. $3 \mathrm{~A})$. Para D el mayor promedio (1.84 $\pm 1.0)$ se presentó en la estación $5 \mathrm{y}$ el menor $(1.28 \pm 0.88)$ en la 8 (Fig. 3B). El índice J' tuvo el valor promedio más alto $(0.90 \pm 0.12)$ y más bajo $(0.58 \pm 0.45)$ en las estaciones 2 y 3 , respectivamente (Fig. 3C). El análisis de varianza no mostró diferencias significativas entre los sitios de muestreo $(P>0.05)$.

En escala temporal, el valor promedio más alto de diversidad $\left(\mathrm{H}^{\prime}\right.$ $=1.51 \pm 0.26)$ se presentó en enero (Fig. 3D) y el más bajo $(0.94 \pm 0.60)$ en marzo, sin diferencias significativas entre meses $(P>0.05)$. La riqueza promedio mayor $(\mathrm{D}=2.02 \pm 0.29) \mathrm{se}$ registró en enero (Fig. 3E) y la menor $(\mathrm{D}=0.97 \pm 0.85)$ en noviembre. Se encontraron diferencias significativas para D entre meses (ANOVA-F $(5,42)$ $=3.55, P=0.0091)$. Las equitatividades promedio más altas $\left(\mathrm{J}^{\prime}=0.92\right.$ \pm 0.13 y J' $=0.92 \pm 0.07)$ se mostraron en mayo y enero, respectivamente (Fig. 3F), y la más baja ( $\mathrm{J}^{\prime}=0.57 \pm$ $0.37)$ en noviembre con diferencias entre meses (Kruskal-Wallis-H $(5,48)$ $=15.37, P=0.0089)$. El análisis de varianza no indicó diferencias significativas entre temporadas del año para los índices de diversidad $(P>0.05)$. Los valores espacio-temporales totales para estos índices fueron: $\mathrm{H}^{\prime}=$ $2.7, \mathrm{D}=5.0 \mathrm{y} \mathrm{J}=0.77$. 

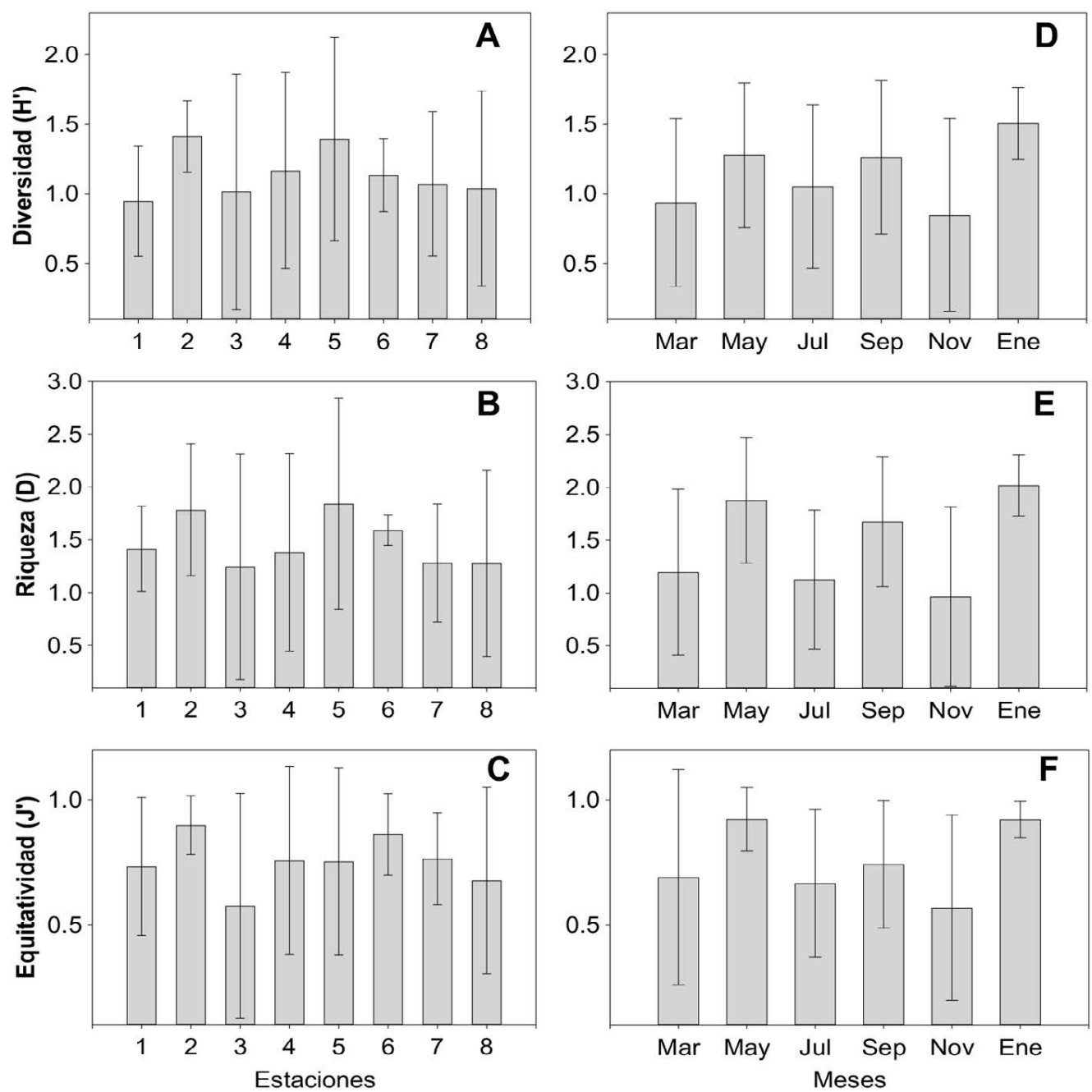

Fig. 3. Valores promedio espaciales (A, B y C) y temporales (D, E y F) ( $\pm 1 \mathrm{DE})$ de los índices de diversidad H', D y J' para la comunidad de peces en el sistema lagunar del Ostión

Fig. 3. Spatial (A, B, and C) and temporal (D, E, and F) average values ( \pm 1 SD) of the diversity indices H', D, and J' for the fish community in the Ostión Lagoon System 
El comportamiento de la den- la estación 1 y los más bajos $(0.007$ sidad (ind. $\left./ \mathrm{m}^{2}\right)$, la biomasa $\left(\mathrm{g} / \mathrm{m}^{2}\right)$ y ind. $\left./ \mathrm{m}^{2} \pm 0.006\right)$ en las estaciones $3 \mathrm{y}$ el peso promedio (g/ind.) no reflejó 6 (Fig. 4A). Las especies que presencambios marcados en el gradiente taron mayor densidad fueron $C$. spiespacial (Fig. 4). De esta manera, el lopterus y D. rhombeus. La biomasa valor promedio más alto en densidad (0.019 ind. $\left./ \mathrm{m}^{2} \pm 0.023\right)$ se registró en promedio varió de $0.41 \mathrm{~g} / \mathrm{m}^{2} \pm 0.37$, en la estación 4 , a $0.19 \mathrm{~g} / \mathrm{m}^{2} \pm 0.19$, en
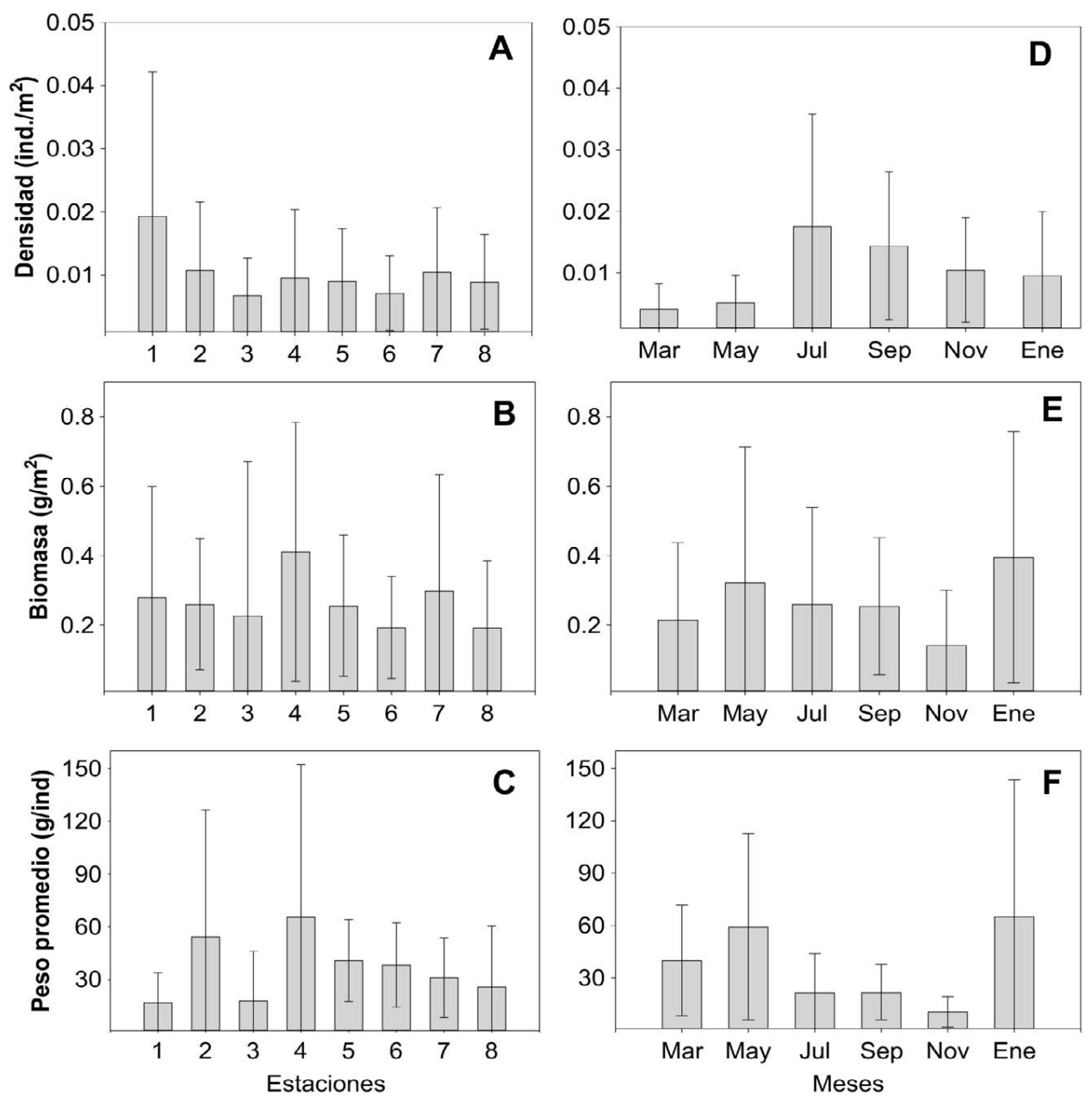

Fig. 4. Valores promedio espaciales $(\mathrm{A}, \mathrm{B}$ y $\mathrm{C})$ y temporales $(\mathrm{D}, \mathrm{E}$ y F $)( \pm 1 \mathrm{DE})$ en densidad, biomasa y peso promedio para la comunidad de peces en el sistema lagunar del Ostión Fig. 4. Spatial (A, B, and C) and temporal (D, E, and F) average values ( \pm 1 SD) in density, biomass, and average weight for the fish community in the Ostión Lagoon System 
la 8 (Fig. 4B). Las especies Opsanus beta, Cathorops aguadulce y Archosargus probatochepalus manifestaron la biomasa más alta. El peso promedio de los peces evidenció su mayor valor en la estación 4 (65.61 g/ind. \pm 86.67) y el menor en la $1(16.93 \mathrm{~g} /$ ind. \pm 16.91) (Fig. 4C). Las especies con mayor peso promedio fueron $G$. micrura, Hypanus americana y O. niloticus. No se encontraron diferencias significativas entre estaciones para las variables señaladas $(P>0.05)$.

En escala temporal, el valor promedio más alto en densidad $(0.018$ ind. $\left./ \mathrm{m}^{2} \pm 0.02\right)$ se registró en julio $\mathrm{y}$ el más bajo $\left(0.004\right.$ ind. $\left./ \mathrm{m}^{2} \pm 0.004\right)$ en marzo (Fig. 4D). La biomasa (Fig. 4E) $\mathrm{y}$ el peso promedio (Fig. 4F) fueron mayores en enero $\left(0.40 \mathrm{~g} / \mathrm{m}^{2} \pm 0.36\right.$ y $65.15 \mathrm{~g}$ /ind. \pm 78.18$)$ y menores en noviembre $\left(0.14 \mathrm{~g} / \mathrm{m}^{2} \pm 0.16\right.$ y 10.64 $\mathrm{g} /$ ind. \pm 8.72$)$, sin diferencias significativas entre meses $(P>0.05)$. Entre temporadas del año, solo se observaron diferencias significativas para la densidad (Kruskal-Wallis-H $(2,48)=$ $10.088, P=0.006$ ), con los valores promedio más bajos en secas y más altos en lluvias. Los valores espacio-temporales totales para estos parámetros fueron: ind $. / \mathrm{m}^{2}=0.010, \mathrm{~g} / \mathrm{m}^{2}=0.265 \mathrm{y} \mathrm{g} /$ ind. $=25.89$.

El índice del valor de importancia (IVI) indicó que $C$. spilopterus, $O$. beta, D. rhombeus y $C$. aguadulce fueron dominantes en el sistema, las cuales representaron el $52 \%$ en número y el $45 \%$ en peso de la captura total. $C$. spilopterus y $O$. beta presentaron el valor más alto de IVI con los mayores porcentajes en número y peso, respectivamente (Cuadro 2). De acuerdo con la clasificación de componentes ícticos, 25 especies $(73.5 \%)$ fueron marinas eurihalinas, cinco (14.7\%) marinas estenohalinas, tres $(8.8 \%)$ estuarinas y una $(2.9 \%)$ dulceacuícola. También se observó que 27 especies $(79.4 \%)$ se asocian siempre con el fondo, cinco (14.7\%) con el fondo y media agua y dos $(5.9 \%)$ con media agua y superficie. Asimismo, fueron identificadas cuatro categorías tróficas en el sistema, de las cuales destacaron 25 especies carnívoras (73.5\%), como C. spilopterus, $O$. beta y $C$. aguadulce que son dominantes; cinco omnívoras (14.7\%) representadas por A. probatocephalus y $D$. rhombeus, esta última también dominante; dos especies detritívoras (5.9\%) como M. curema y O. niloticus, y dos planctívoras (5.9\%), A. clupeoides y $A$. mitchilli, las cuales fueron categorías tróficas poco abundantes.

El diagrama de ordenación (Fig. 5) muestra la correspondencia entre la abundancia de las especies, las estaciones y las variables ambientales, así como la importancia de estas en la composición y distribución de los peces en la comunidad. El ACC mostró que la relación entre las especies y las variables ambientales fue explicada en un $46 \%$ de la varianza total con $28.5 \%$ (autovalor $=0.28$ ) para 


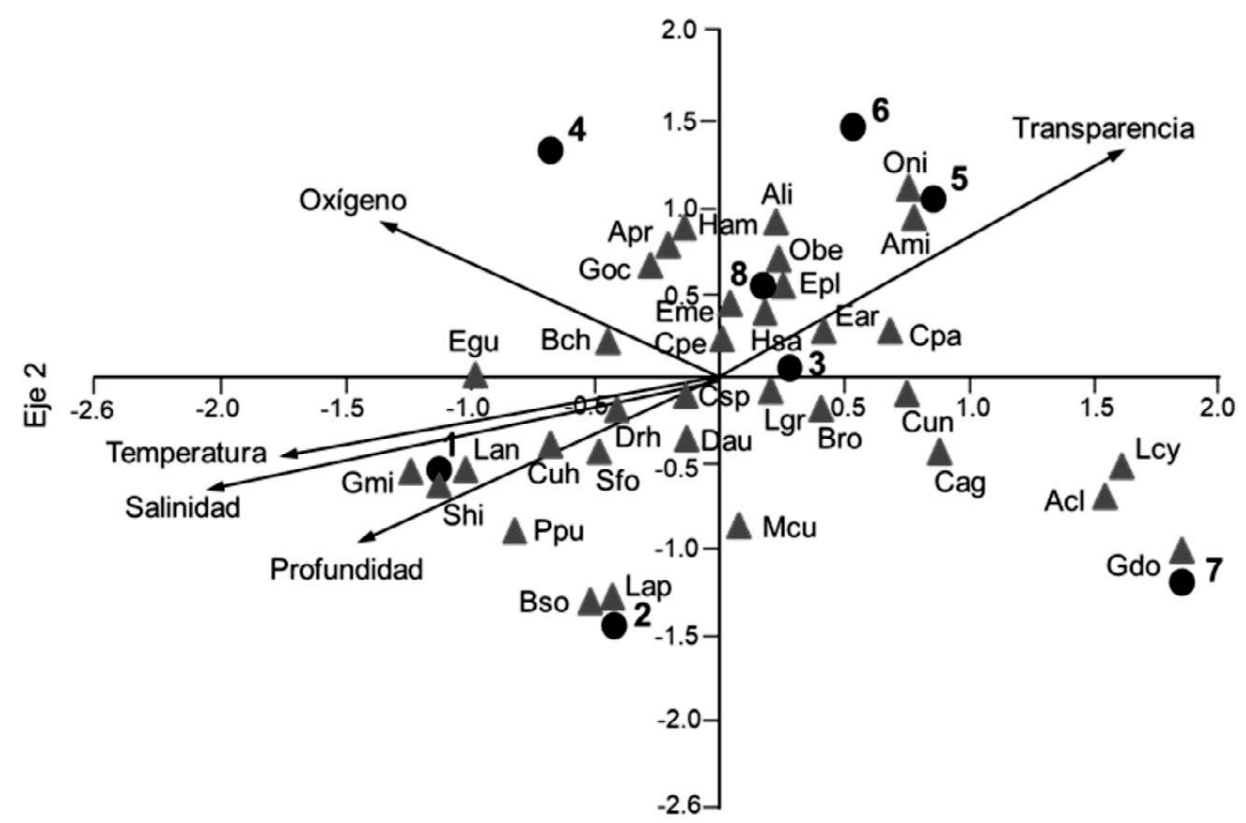

Eje 1

Fig. 5. Diagrama del análisis de correspondencias canónicas de la abundancia de las especies, las variables ambientales y las estaciones de muestreo en el sistema lagunar del Ostión. La longitud y dirección de las flechas indican la importancia relativa de las variables ambientales. Las abreviaturas de las especies (AB) se indican en Cuadro 2.

Fig. 5. Canonical Correspondence Analysis Plot of species abundance, environmental variables, and sampling stations in the Ostión Lagoon System. Arrow length and direction indicate the relative importance of environmental variables. Species abbreviations (AB) are listed in Table 2 .

el eje 1 y $17.5 \%$ (autovalor $=0.17$ ) para el 2. Para el eje 1, las mayores correlaciones de las variables ambientales fueron: salinidad $(r=-0.89)$, temperatura $(\mathrm{r}=-0.78)$, profundidad $(\mathrm{r}=-0.68)$ y transparencia $(\mathrm{r}=0.80)$ y para el 2 , el oxígeno disuelto $(\mathrm{r}=$ $0.57)$ y la transparencia $(\mathrm{r}=0.53)$. Un grupo de especies con distribución restringida y abundancias muy bajas se asoció con la estación 1 (eje 1), estas correspondieron al componente estenohalino, como G. micrura (Gmi) y S. hispidus (Shi), y al eurihalino, como L. analis (Lan), C. uhleri (Cuh) y $S$. foetens (Sfo); asimismo, con la estación 2 (eje 2) e igualmente eurihalinas, como L. apodus (Lap), $B$. soporator (Bso) y P. punctatus (Ppu). En estas dos estaciones, se registraron los promedios más altos de salinidad, temperatura y profundidad de 
la laguna (Cuadro 1). En la estación 3 (eje 1), se distribuyeron otras especies eurihalinas con abundancias bajas, como E. argenteus (Ear), C. parallelus (Cpa) y L. griseus (Lgr), y estuarinas, como H. sabinus (Hsa), vinculadas con mayor transparencia del agua. Enlazadas a la estación 4 (eje 2) se presentaron especies estenohalinas con mayor abundancia y valores altos de oxígeno, como $H$. americana (Ham) y G. oceanicus (Goc), y eurihalinas, como A. probatochepalus. Otras especies eurihalinas asociadas a las estaciones 5 y 7 (eje 1) solo se distribuyeron en estos sitios con baja abundancia, como A. mitchilli (Ami), L. cyanopterus (Lcy) y A. clupeoides (Acl), así como estuarinas, G. dormitor (Gdo), y dulceacuícolas, como O. niloticus (Oni). En las estaciones 6 y 8 (eje 2), se presentaron especies eurihalinas con distribuciones más amplias y abundancias mayores en el sistema, como A. lineatus (Ali), E. plumieri (Epl) y E. melanpterus (Eme). Las especies dominantes del sistema se distribuyeron en toda la laguna, no obstante, la mayor abundancia de C. spilopterus y D. rhombeus se localizó hacia la mitad norte, donde son mayores la salinidad, temperatura y profundidad, mientras que $C$. aguadulce y $O$. beta, hacia la mitad sur, con menores valores de las variables mencionadas, incluyendo transparencia y profundidad.

\section{DISCUSIÓN}

La Laguna del Ostión presenta una variación espacio-temporal de los parámetros fisicoquímicos, relacionada con la entrada de agua marina del golfo de México, a través de la boca de conexión y por la descarga de agua dulce de los ríos principales del sistema. Estos procesos están determinados por las temporadas climáticas, la superficie, forma y profundidad del sistema, así como por la amplitud de la marea, entre otros (Snedden et al. 2013; Sánchez-Gil et al. 2019). La salinidad fue más alta hacia la boca de conexión marina y más baja hacia la cabecera del sistema con influencia fluvial. Este gradiente salino fue descrito de forma preliminar por Bozada \& Chávez (1986), con valores registrados de 0 a 38 UPS; en el presente estudio no se anotaron valores cero de salinidad en ninguno de los sitios monitoreados. El comportamiento temporal de la salinidad estuvo estrechamente ligado a la estacionalidad climática, con los valores más altos en mayo (secas), como consecuencia de la menor precipitación (37.2 mm, CNA-SMN, 2020) y mayor efecto de la marea hacia el interior del sistema. Durante la época de lluvias, la salinidad fue menor como resultado de la mayor precipitación (363 mm, CNA-SMN, 2020) y la descarga de los ríos, como ocurrió en septiembre (Lara-Domínguez et 
al. 2008). En el estudio de Bozada \& Chávez (1986), la salinidad más alta (38 UPS) también se registró en marzo de 1980 (secas), en la boca de conexión marina, y la más baja (0 UPS) en septiembre de 1980 (lluvias), en casi todo el sistema.

La temperatura del agua presentó poca variación entre sitios en el sistema. Esta fue ligeramente mayor para las estaciones de la zona norte, debido a la entrada de agua marina más caliente por efecto de la marea, mientras que en la parte sur de la laguna fue menor, dado el drenaje de aguas más frías de los ríos. Otro factor que mantuvo los valores de temperatura con poca variación espacial fue la escasa profundidad que presenta el sistema y buena mezcla vertical (Brucet et al. 2012; Day et al. 2013), por lo cual, la diferencia entre sitios fue pequeña $\left(0.62^{\circ} \mathrm{C}\right)$ y no significativa. A lo largo del año la temperatura del agua se relacionó con el cambio térmico del aire de las épocas climáticas, lo cual se reflejó en el cuerpo lagunar, al presentar los valores más altos en julio (lluvias) y los más bajos en enero (nortes) (Bozada \& Chávez, 1986; Díaz-Ruiz et al. 2008), hecho que ocurre en otros ecosistemas costeros similares (Díaz-Ruiz et al. 2012; Aguirre-León et al. 2018; Sánchez-Gil et al. 2019).

El oxígeno disuelto presentó poca variación espacial, no obstante, los valores más altos se registraron en la zona norte del sistema, debido a los procesos de mezcla por los vientos locales y corrientes que ocurren en las cercanías de la boca con el mar, así como por la vegetación sumergida $(R$. maritima, $S$. filiforme) en áreas someras (Díaz-Ruiz et al. 2008); además, este parámetro puede favorecer o limitar la presencia o ausencia de peces en los sistemas acuáticos costeros (Craig, 2012; Díaz-Ruiz et al. 2018). Temporalmente, el oxígeno disuelto mostró un patrón relacionado con las variaciones térmicas entre periodos climáticos y con la frecuencia e intensidad de los eventos norte que se presentan en la región. La mayor concentración se registró en noviembre y enero, enlazada a bajas temperaturas $y$ acción del viento de esta época, mientras que los valores más bajos se dieron en mayo (secas), debidos al incremento de la temperatura del agua y al aporte de materia orgánica por los ríos durante esta última temporada, tal como ocurre en otros sistemas lagunares (Aguirre-León et al. 2010; Sánchez-Gil et al. 2019).

En este trabajo, la profundidad y la transparencia dejaron ver una tendencia espacial similar con los valores más altos hacia la boca de conexión y los más bajos en la cabecera de la laguna. Este comportamiento está asociado al régimen de precipitación pluvial, a la geomorfología del sistema, a la variación de la marea (Pérez-Ruzafa et al. 2007). Durante el año, estos parámetros fueron influenciados por la intensidad 
de las lluvias y los vientos del norte. En esta última época, la mayor profundidad se debió al incremento del caudal de los ríos en la zona sur y a las precipitaciones de septiembre en el sistema, mientras que la menor se presentó al final de las lluvias e inicio de la temporada de nortes. La transparencia reveló un comportamiento relacionado con la profundidad, con valores altos en enero (nortes) y bajos en septiembre (lluvias). El comportamiento anterior también ha sido observado en sistemas costeros similares (Lara-Domínguez et al. 2008; Hutchinson et al. 2014; Aguirre-León et al. 2018). Otro factor que afecta las variaciones de la profundidad-transparencia es el azolve del sistema, lo estrecho de su boca marina, la erosión de sus riveras por presión agropecuaria y demográfica (Lara-Domínguez et al. 2009; López-Portillo et al. 2017).

La comunidad de peces en la Laguna del Ostión es la menos estudiada de Veracruz en las últimas tres décadas. La mayoría de la literatura sobre este sistema solo integra elencos sistemáticos, sin ningún análisis ecológico adicional y mucho menos sobre los cambios en la riqueza de especies en escala espacio-temporal, por lo que no es posible realizar un examen retrospectivo completo acerca de las variaciones del número de especies de la ictiofauna en esta laguna. Los estudios previos no indican las artes de pesca que fueron utilizadas, (excepto Bozada \& Chávez, 1986), cada una de las cuales arroja diferentes composiciones específicas, por lo que es razonable suponer que el sistema puede contener especies no registradas. En esta investigación, se identificaron 34 especies, de las cuales 12 son registros nuevos para el área. Existen registros históricos de la riqueza de especies para la Laguna del Ostión realizados por Bozada \& Chávez (1986) con 36 especies, Villanueva et al. (1988) con 3, Lozano-Vilano et al. (1993) con 3, Díaz-Ruiz et al. (2008) con 12 y Lara-Domínguez et al. (2008) con 14, gracias a los que el total asciende a 68 especies, en comparación con las 62 documentadas por Lara-Domínguez et al. (2011). De acuerdo con este análisis, al parecer transcurrieron, entre estos registros, 22 años sin recolectas nuevas. Cabe mencionar la presencia de $O$. niloticus como especie exótica dulceacuícola registrada por Díaz-Ruiz et al. (2008) y, recientemente, del pez diablo Pterygoplichthys spp. de distribución restringida en la zona suroeste de la laguna, en áreas de influencia de los ríos Temoloapan y Huazuntlán (PNUD, 2017). La existencia de estos peces invasores podría incrementar el elenco de la Laguna del Ostión, tan pronto se identifiquen con precisión cuántas especies de pez diablo se encuentran. Por otra parte, los resultados de las curvas de acumulación de especies representaron el número de estas capturadas en cada sitio de muestreo, lo cual valida el esfuerzo de pesca utilizado en este 
estudio. Estos datos sugirieron que la Laguna del Ostión puede tener mayor riqueza de especies, por lo que se considera que un esfuerzo adicional estandarizado en espacio y tiempo en esta laguna resultaría en la captura de otras especies.

En la Laguna del Ostión, se observó que los cambios en los valores de diversidad entre los diferentes ambientes están estrechamente relacionados con las características propias de cada uno de estos, siendo más altos hacia el canal de comunicación marino y en la parte media del sistema, donde es más marcada la influencia mareal, así como son mayores la salinidad y la temperatura. Estas condiciones determinaron la entrada y abundancia proporcional de especies que utilizan dicho canal como, C. spilopterus, D. rhombeus, $H$. americana, G. oceanicus y O. beta. Se ha notado un comportamiento similar en las lagunas Chica-Grande al norte y en Sontecomapan al sur de Veracruz, las cuales presentan canales sinuosos de marea, lo que favorece el ingreso de especies estenohalinas hacia estos y eurihalinas en todo el sistema (Aguirre-León et al. 2014; Aguirre-León et al. 2018). La profundidad, transparencia y descarga de agua dulce asociadas a la precipitación también tienen un efecto en la distribución y riqueza de los peces (e. g. C. aguadulce y O. niloticus), como se vio en la parte sur del sistema, donde hubo baja salinidad, transparencia y profundidad (Barletta et al. 2005;
Greenwood et al. 2007; Díaz-Ruiz et al. 2018). La diversidad y riqueza tuvieron valores más altos en enero (nortes), ya que se registraron especies eurihalinas y estenohalinas del componente marino con mayor abundancia durante este mes, lo cual reflejó que utilizan distintos ambientes de la laguna como áreas de protección. Mientras, los valores más bajos de estos parámetros se vinculan a salinidades altas en marzo (secas) y bajas en noviembre (nortes), ligados a las variaciones estacionales de salinidad y temperatura que condicionan la migración de las especies al interior del sistema lagunar. Estas migraciones además coinciden con periodos de alta productividad y mayor disponibilidad de alimento en el sistema, que son optimizados por las especies de peces durante sus ciclos de vida (Cowan et al. 2013), como lo visto en el presente estudio.

El comportamiento de la abundancia de los peces está influenciado por las variaciones hidrológicas de los sistemas durante sus ciclos de vida (Miller \& Skilleter, 2006; Arceo-Carranza et al. 2010). En la Laguna del Ostión, la densidad y la biomasa estuvieron relacionadas con los gradientes de salinidad, temperatura y profundidad. Así, la mayor abundancia numérica de peces se registró en zonas cercanas a la boca marina, debido al desplazamiento, del mar hacia la laguna, de especies eurihalinas (como juveniles y preadultos) para alimentarse y crecer durante la época de lluvias 
(julio y septiembre) y nortes (enero), como C. spilopterus, D. rhombeus, E. gula, E. melanopterus y $S$. foetens. En la parte media y sur del sistema, la mayor biomasa fue por la presencia de individuos de más talla del componente estuarino, eurihalino y dulceacuícola, como C. aguadulce, A. probatocephalus, C. parallelus, $H$. sabinus, O. beta y $O$. niloticus, que utilizan el sistema para alimentarse, madurar o protegerse durante nortes-secas (enero-marzo). Variaciones similares en la abundancia de estos peces, ligadas a las condiciones ambientales del sistema, también han sido observadas en otros sistemas costeros tropicales (Castillo-Rivera et al. 2010; Sosa-Pérez et al. 2012). En el Ostión, algunas de las especies mencionadas son consideradas dominantes y otras tienen importancia comercial, como los robalos Centropomus spp., sin embargo, su conocimiento biológico y ecológico es escaso en este sistema, por lo que es necesario incrementarlo en un futuro cercano.

El análisis de la dominancia es un aspecto fundamental en las comunidades de peces, ya que permite definir la trascendencia ecológica de las especies, a través de las interacciones establecidas entre ellas y con los ambientes que utilizan a lo largo de su ciclo de vida. Las especies dominantes regulan los flujos estacionales de abundancia en el sistema, por lo que son fundamentales en el mantenimiento de la diversidad en el ecosistema (Sheridan
\& Minello, 2003; Yáñez-Arancibia \& Day, 2010; Sánchez-Gil et al. 2019). En la Laguna del Ostión, la relación entre abundancia, peso, frecuencia y distribución de los peces con los gradientes ambientales del sistema determinó que $C$. spilopterus, $D$. rhombeus, $C$. aguadulce y $O$. beta fueron dominantes en el sistema lagunar, en todas las épocas climáticas. Estas especies han desarrollado estrategias biológicas de crianza, protección, alimentación y reproducción, que les han permitido mantenerse como predominantes en el sistema. En comparación, Bozada \& Chávez (1986) reportaron a $D$. rhombeus como la más abundante y con menor proporción a E. melanopterus y Brevoortia patronus. Estudios ictiológicos en otros sistemas lagunares del golfo de México (Aguirre-León et al. 2006; Aguirre-León et al. 2010; Arceo-Carranza et al. 2013) han registrado a las especies previamente mencionadas o afines como dominantes o abundantes en diferentes magnitudes.

En los sistemas costeros, los peces han desarrollado adaptaciones fisiológicas que les dejan usar los gradientes ambientales y optimizar los hábitats tanto lagunares como marinos de estos sistemas, durante diferentes etapas de sus ciclos de vida, por lo que se reconocen varios componentes ícticos (Yáñez-Arancibia et al. 1985; Castro-Aguirre et al. 1999; Able, 2005; Cowan et al. 2013). Esto se vio para las especies estenohalinas 
que solo ingresan a los ambientes con menor cambio salino y son poco frecuentes o raras (Díaz-Ruiz et al. 2004; Brucet et al. 2012; França et al. 2012), como G. micrura. L. analis y $S$. hispidus, presentes en la cercanía de la boca marina y el canal de comunicación. Los componentes eurihalinos con gran representación tanto en especies como en número de individuos contribuyeron con alta abundancia numérica en el sistema, tal es el caso de C. spilopterus. Especies estuarinas como $C$. aguadulce permanecen todo su ciclo de vida en la laguna y optimizan los gradientes ambientales. Mientras, O. niloticus, como especie dulceacuícola, presentó distribución restringida en zonas de influencia fluvial. Para otros sistemas lagunares como Alvarado, Chica-Grande, La Mancha y Sontecomapan en Veracruz, se registró, igualmente, mayor proporción de componentes eurihalinos y menor tanto de estenohalinos como de estuarinos (Franco-López et al. 2012; Aguirre-León et al. 2018; Díaz-Ruiz et al. 2018).

No existen estudios publicados a la fecha sobre la dieta y los hábitos alimentarios de los peces en la Laguna del Ostión, por lo que son escasos los datos sobre la composición de grupos tróficos de la ictiofauna en el sistema. El estudio de la ecología trófica en peces faculta conocer y comprender aspectos sobre la biología, la estructura ecológica y el papel funcional de las especies o conjuntos de especies en estos sistemas costeros (Hagy \& Kemp, 2013; Abrantes et al. 2015). En esta indagación, se observó que las especies carnívoras $C$. spilopterus, O. beta, C. parallelus y L. griseus se distribuyeron en áreas de pastos y raíces de manglar, donde son abundantes los crustáceos, mientras que la mayor abundancia de omnívoros y detritívoros, como D. rhombeus, $S$. hispidus, M. curema y O. niloticus, se registró en la parte central y sur de la laguna. Los planctívoros A. clupeoides y $A$. mitchilli fueron poco abundantes, pero frecuentes en áreas con influencia marina. Su comportamiento trófico depende de la variedad de las adaptaciones morfológicas que los peces presenten para su alimentación a través de su ontogenia, así como de la disponibilidad del sustento en los hábitats que utilizan y de las interacciones ecológicas de las especies con el ecosistema (Zarate-Hernández et al. 2007; Engdaw et al. 2013; Arceo-Carranza et al. 2013; Clark \& Pessanha, 2014).

El análisis del vínculo composición-abundancia de los peces con sus hábitats permitió identificar la presencia de asociaciones de peces relacionados con los gradientes ambientales del sistema, donde la distribución de las especies estuvo condicionada por el comportamiento ecológico de los diferentes componentes ícticos. De esta manera, la presencia de especies estenohalinas y eurihalinas estuvo asociada con zonas de mayor influencia 
marina cercanas a la boca de conexión $\mathrm{y}$ al canal de comunicación del sistema, como E. gula, L. analis, L. apodus, G. micrura, G. oceanicus, P. punctatus y $S$. hispidus. Estas se distribuyeron en áreas con vegetación sumergida y de manglar, así como con valores mayores de oxígeno disuelto, transparencia y profundidad, observando un alto porcentaje de especies que se vinculan con el fondo. Asimismo, el conjunto de especies que estuvo estrechamente relacionado con mayores cambios de salinidad durante los meses de estudio, se caracterizó por peces estuarinos y eurihalinos, como C. parallelus, $C$. aguadulce y $G$. dormitor, los cuales toleran condiciones de baja salinidad, menor profundidad y transparencia en áreas de influencia fluvial. Las especies de estos conjuntos dependen del sistema para protección, alimentación y crecimiento, durante las temporadas climáticas del año. Un comportamiento similar al que anteriormente se señala ha sido documentado en otros sistemas costeros tropicales (Vaslet et al. 2010; Aguirre-León et al. 2014; Romero-Berny et al. 2020).

El presente estudio contribuye al conocimiento de la estructura ecológica de la comunidad de peces en la Laguna del Ostión, donde son evidentes la carencia informativa científica y un adecuado programa de monitoreo para entender el estado de conservación del sistema. De esta manera, el análisis derivado de este trabajo constituye un marco de referencia para evaluaciones futuras de comparación ambiental y ecológica de la dinámica comunitaria de peces del sistema, sobre todo por su ubicación cercana al puerto de Coatzacoalcos al sur del estado de Veracruz, el cual sigue presentando, en la actualidad, un alto desarrollo industrial y urbano.

\section{AGRADECIMIENTOS}

Al Departamento de Hidrobiología de la Universidad Autónoma Metropolitana Iztapalapa, a través del proyecto "Caracterización Ecológica de los Ambientes Costeros Mexicanos" (UAMI-CBS, 2018-2020), y al CONACyT-(FOMIX) proyecto C0137014, por el apoyo institucional y económico para realizar este estudio. Los autores agradecen a todos los estudiantes su colaboración en las campañas de campo y actividades de laboratorio, en particular, a Mayra Juárez Bautista. Se agradece, también, a los árbitros sus comentarios y sugerencias realizadas. 


\section{REFERENCIAS}

Able, K. W. (2005). A re-examination of fish estuarine dependence: Evidence for connectivity between estuarine and ocean habitats. Estuar. Coast. Shelf Scie., 64, 5-17. https://doi.or$\mathrm{g} / 10.1016 /$ j.ecss.2005.02.002

Abrantes, K. G., Barnett, A., Baker, R. \& Sheaves, M. (2015). Habitat-specific food webs and trophic interactions supporting coastal-dependent fishery species: an Australian case study. Rev. Fish Biol. Fish., 25, 337-363. https:// doi.org/10.1007/s11160-015-9385-y

Aguirre-León, A. \& Díaz-Ruiz, S. (2006). Estructura de tallas, madurez gonádica y alimentación del pez Diapterus rhombeus (Gerreidae) en el sistema fluvio-deltaico Pom-Atasta, Campeche, México. Rev. Biol. Trop., 54(2), 599-611. https://doi.org/10.15517/rbt. v54i2.13927

Aguirre-León, A., Díaz-Ruiz, S. \& Ramírez-Huerta, A. (2010). Ecología de peces dominantes costeros tropicales. Estudio para el sistema fluvio-deltaico Pom- Atasta, Campeche. Serie Académicos 96. México: CBS, UAM-Xochimilco.

Aguirre-León, A., Pérez-Ponce, H. E. \& Díaz-Ruiz, S. (2014). Heterogeneidad ambiental y su relación con la diversidad y abundancia de la comunidad de peces en un sistema costero del Golfo de México. Rev. Biol. Trop., 62(1), 145-163. https://doi.org/10.15517/rbt. v62i1.8212

Aguirre-León, A., Díaz-Ruiz, S. \& Guillén-Castrillo, M. (2018). Fish community structure and its relationship with environmental behavior in Sontecomapan Lagoon, Los Tuxtlas Biosphere Reserve, Veracruz, Mexico. In M. E. Castellanos-Páez, A. Esquivel, J.
Aldeco-Ramírez \& M. Pagano (Eds.), Ecology of the Sontecomapan Lagoon, Veracruz (pp. 205-234). Mexico-France: UAM-X/IRD.

Arceo-Carranza, D., Vega-Cendejas, M. E., Montero-Muñoz, J. L. \& Hernández de Santillana, M. (2010). Influencia del hábitat en las asociaciones nictimerales de peces en una laguna costera tropical. Rev. Mex. Biodiver, 81, 823-837.

Arceo-Carranza, D., Vega-Cendejas, M. E. \& Hernández de Santillana, M. (2013). Day and night trophic variations of dominant fish species in a lagoon influenced by freshwater seeps. J. Fish Biol., 82(3), 54-68. https://doi.org/10.22201/ ib.20078706e.2010.003.652

Baltz, D. \& Yáñez-Arancibia, A. (2013). Ecosystem-based management of coastal fisheries in the Gulf of Mexico: environmental and anthropogenic impacts and essential habitat protection. In J. W. Day \& A. Yáñez-Arancibia (Eds.), The Gulf of Mexico origin, waters, and biota (pp. 337-370). EE. UU.: Texas A \& M University Press.

Barletta, M., Barletta-Bergan, A., SaintPaul, U. \& Hubold, G. (2005). The role of salinity in structuring the fish assemblages in a tropical estuary. $J$. Fish Biol., 66(1), 45-72. https://doi. org/10.1111/j.0022-1112.2005.00582.x

Beltrán-García, F. de M., Deita, S., Domínguez-Moctezuma, M., Pérez-Lugo, E. \& Reyes-Díaz, M. (2019). ¿Qué ha pasado con las pesquerías en el estado de Veracruz en los últimos 25 años? En F. Lango-Reynoso, A. V. Botello \& M. R. Castañeda-Chávez (Eds.), Temas selectos de vulnerabilidad costera en el estado de Veracruz (pp. 127-143). México: Universidad Autónoma de Campeche. Instituto de Ecología, Pesquerías y Oceanografía del Golfo de México (EPOMEX). 
Bozada, L. \& Chávez, F. (1986). La fauna acuática de la Laguna del Ostión. Serie Medio Ambiente en Coatzacoalcos. Centro de Ecodesarrollo, Universidad Veracruzana. México: Ingramex, S. A.

Brucet, S., Boix, D., Nathansen, L. W., Quintana, X. D., Jensen, E., Balayla, D., Meerhoff, M. \& Jeppesen, E. (2012). Effects of temperature, salinity and fish in structuring the macroinvertebrate community in shallow lakes: implications for effects of climate change. PLoS One, 7, 1-11. https://doi. org/10.1371/journal.pone.0030877

Castillo-Rivera, M., Zárate-Hernández, R., Ortiz-Burgos, S. \& Zavala-Hurtado, A. (2010). Diel and seasonal variability in the fish community structure of a mud bottom estuarine habitat in the Gulf of Mexico. Mar. Ecol., 31(4), 633-642. https://doi. org/10.1111/j.1439-0485.2010.00394.x

Castro-Aguirre, J. L., Espinoza-Pérez, H. \& Schmitter-Soto, J. J. (1999). Ictiofauna estuarino-lagunar y vicaria de México. México: Limusa.

Chi-Espínola, A. \& Vega-Cendejas, M. E. (2016). Food resources of Eucinostomus (Perciformes: Gerreidae) in a hyperhaline lagoon: Yucatan Peninsula, Mexico. Rev. Biol. Mar. Oceanog., 51(2), 395-406. https://doi.org/10.4067/ S0718-19572016000200016

Clark, F. J. \& Pessanha, A. L. (2014). Diet and ontogenetic shift in habitat use by Rhinosardinia bahiensis in a tropical semi-arid estuary, north-eastern Brazil. J. Mar. Biol. Assoc. United Kingdom, 95(1), 175-183. https://doi. org/10.1017/S0025315414000939

Clarke, K. R. \& Warwick, R. M. (2006). Change in marine communities: An approach to statistical Analysis and interpretation. United Kingdom: Primer-E. Plymouth.
CNA-SMN. (Comisión Nacional del Agua-Sistema Meteorológico Nacional). (2020). Información Estadística Climatológica. http://smn.conagua.gob.mx.

Cowan, J. H., Yáñez-Arancibia, A., Sánchez-Gil, P. \& Deegan, L. (2013). Estuarine nekton. In J. W. Day, B. C. Crump, W. M. Kemp \& A. Yáñez-Arancibia (Eds.), Estuarine ecology (pp. 327-355). EE. UU.: Willey \& Sons. https://doi. org/10.1002/9781118412787.ch13

Craig, J. K. (2012). Aggregation on the edge: effects of hypoxia avoidance on the spatial distribution of brown shrimp and demersal fishes in the Northern Gulf of Mexico. Mar. Ecol. Prog. Ser., 445, 7595. https://doi.org/10.3354/meps09437

Day, J. W., Yáñez-Arancibia, A., Kemp, W. M. \& Crump, B. C. (2013). Introduction to estuarine ecology. In J. W. Day, B. C. Crump, W. M. Kemp \& A. Yáñez-Arancibia (Eds.), Estuarine ecology (pp. 1-18). EE. UU.: Willey \& Sons. https:// doi.org/10.1002/9781118412787.ch1

De la Cruz-Agüero, G. (1994). ANACOM Sistema para el análisis de comunidades. Versión 3.0. México: CICIMAR-IPN.

Díaz-Ruiz, S., Cano-Quiroga, E., Aguirre-León, A. \& Ortega-Bernal, R. (2004). Diversidad, abundancia $\mathrm{y}$ conjuntos ictiofaunísticos del sistema lagunar-estuarino Chantuto-Panzacola, Chiapas, México. Rev. Biol. Trop., 52(1), 187-199. https://doi. org/10.15517/rbt.v52i1.14879

Díaz-Ruiz, S., Aguirre-León, A., Juárez, M., Matsumoto, J. \& Hernández, J. (2008). Evaluación ecológica de las comunidades de peces: Laguna La Mancha y Laguna el Ostión, Veracruz Golfo de México. (Reporte Final-CONACYT-FOMIX). México: Instituto de Ecología y Universidad Autónoma Metropolitana. 
Díaz-Ruiz, S., Aguirre-León, A., Calva-Benítez, L. G. \& Barba-Macías, E. (2012). Comportamiento ambiental y estructura comunitaria de peces en el sistema Carretas-Pereyra, Reserva de la Biosfera La Encrucijada, Chiapas. En A. J. Sánchez, X. Chiappa-Carrara \& R. Brito-Pérez (Eds.), Recursos acuáticos costeros del sureste (pp. 415-434). México: CONCIYTEY-UNAM.

Díaz-Ruiz, S., Aguirre-León, A., Mendoza-Sánchez, E. \& Lara-Domínguez, A. L. (2018). Factores ambientales que influyen en la ictiofauna de la Laguna La Mancha, sitio Ramsar, Golfo de México. Rev. Biol. Trop., 66(1), 246-265. https://doi.org/10.15517/rbt.v66i1.28495

Fricke, R., Eschmeyer, W. N. \& van der Laan, R. (Eds.). (2020). Eschmeyer's Catalog of Fishes: Genera, Species, References. http://researcharchive.calacademy.org/ research/ichthyology/catalog/fishcatmein.asp

Engdaw, F., Dadebo, E. \& Nagappan, R. (2013). Morphometric relationships and feeding habits of Nile Tilapia Oreochromis niloticus (L.) (Pisces: Cichlidae) from Lake Koka, Ethiopia. Inter. J. Fish. Aquat. Scie., 2, 65-71.

Fischer, W. (1995). Species identification sheets for fishery purpose. Western Central Atlantic (Fishing area 31). Rome: FAO.

França, S., Vasconcelos, R. P., Fonseca, V. F., Tanner, S. E., Reis-Santos, P., Costa, M. J. \& Cabral, H. N. (2012). Predicting fish community properties within estuaries: influence of habitat type and other environmental features. Estuar. Coast. Shelf Scie., 107, 22-31. https:// doi.org/10.1016/j.ecss.2012.04.013

Franco-López, J., Abarca-Arenas, L. G., Bedia-Sánchez, C., Barrera-Escoria, H. \& Silva-Contreras, G. (2012). Comportamiento de la comunidad ictiofaunística ante los cambios ambientales a nivel local y regional en la Laguna de Alvarado. En L. F. Del Moral, J. Martínez, J. Franco, A. Ramírez \& J. L. Tello (Eds.), Investigación ictiológica en México (pp. 259-283). México: FES-UNAM-SIMAC.

Froese, R. \& Pauly, D. (Eds.). (2019). FishBase. Version 03. World Wide Web electronic publication. http://www.fishbase.org

García, E. (2004). Modificaciones al sistema de clasificación climática de Köppen. México: Instituto de Geografía, UNAM.

Greenwood, M. F., Matheson, Jr. R. E., McMichael, Jr. R. H. \& MacDonald, T. C. (2007). Community structure of shoreline nekton in the estuarine portion of the Alafia River, Florida: Differences along a salinity gradient and inflow-related changes. Estuar. Coast. Shelf Scie., 74(Issues 1-2), 223-238. https:// doi.org/10.1016/j.ecss.2007.04.010

Hagy, J. D. \& Kemp, W. M. (2013). Estuarine food webs. In J. W. Day, B. C. Crump, W. M. Kemp \& A. Yáñez-Arancibia (Eds.), Estuarine ecology (pp. 417441). EE. UU.: Willey \& Sons. https:// doi.org/10.1002/9781118412787.ch16

Heiman, G. W. (2011). Basic Statistics for the Behavioral Sciences. Sixth Edition. EE. UU.: Wadsworth, Cengage Learning.

Hutchinson, N., Jenkins, G. P., Smith, T. M. \& Brown, A. (2014). Variation with depth in temperate seagrass-associated fish assemblages in southern Victoria, Australia. Estuar. Coast., 37, 801-814. https://doi.org/10.1007/ s12237-013-9742-9

KCS, Inc. (2013). MVSP. Multivariate Statistical Package, Version 3.22. Kovach Computing Services. http://www.kovcomp.com 
Lango-Reynoso, F., Botello, A. V. \& Castañeda-Chávez., M. R. (Eds.) (2019). Temas selectos de vulnerabilidad costera en el estado de Veracruz. México: Universidad Autónoma de Campeche. Instituto de Ecología, Pesquerías y Oceanografía del Golfo de México (EPOMEX).

Lara-Domínguez, A. L., Sáinz-Hernández, E., Landgrave, R., González-Navarro, A. \& Marín-Perea, R. (2008). Evaluación de los recursos naturales y la productividad pesquera de dos lagunas costeras ante el impacto del cambio climático y el ascenso del nivel medio del mar: Laguna de la Mancha y Laguna del Ostión, Veracruz, Golfo de México. México. (Reporte Final-CONACYT-FOMIX). México: Instituto de Ecología y Universidad Autónoma Metropolitana.

Lara-Domínguez, A. L., López-Portillo, J., Ávila-Ángeles, A. \& Vázquez-Lule, A. (2009). Caracterización del sitio de manglar Laguna Ostión. México: Comisión Nacional para el Conocimiento y Uso de la Biodiversidad (CONABIO).

Lara-Domínguez, A. L., Franco-López, J., Bedia-Sánchez, C., Abarca-Arenas, L., Díaz-Ruiz, S., Aguirre-León, A., González-Gándara, C. \& Castillo-Rivera, M. (2011). Diversidad de peces en los ambientes costeros y plataforma continental. En A. Cruz-Angón (Ed.), La biodiversidad en Veracruz: estudio de estado (pp. 505-516). México: CONABIO, UV, INECOL, Gobierno del Estado de Veracruz.

Levene, H. (1960). Robust test for equality on variance. In I. Olkin, S. G. Ghurye, W. Hoeffding, W. G. Madow \& H. B. Mann (Eds.), Contributions of probability and statistics (pp. 278-292). EE. UU.: Stanford University.
López-Portillo, J., Lara-Domínguez, A. L., Vázquez, G. \& Aké-Castillo, J. A. (2017). Water Quality and Mangrove-Derived Tannins in Four Coastal Lagoons from the Gulf of Mexico with Variable Hydrologic Dynamics. J. Coast. Res., 77, 28-38. https://doi. org/10.2112/SI77-004.1

Lozano-Vilano, M. L., García-Ramírez, M. E. \& Contreras-Balderas, S. (1993). Peces costeros y marinos del Estado de Veracruz. En S. I. Salazar-Vallejo \& N. E. González (Eds.), Biodiversidad marina y costera de México (pp. 576-595). México: Comisión Nacional para el Uso y Conocimiento de la Biodiversidad (CONABIO), Centro de Investigaciones de Quintana Roo (CICRO).

Magurran, A. E., Kachonpisitsak, S. \& Ahmad, A. B. (2011). Biological diversity of fish communities: pattern and process. J. Fish Biol., 79(6), 1393-1412. https://doi. org/10.1111/j.1095-8649.2011.03091.x Margalef, R. (1995). Ecología. España: Ediciones Omega.

McGarigal, K., Cushman, S. \& Stafford, S. (2000). Multivariate statistics for wildlife and ecology research. EE. UU.: Springer-Verlag. https://doi. org/10.1007/978-1-4612-1288-1

Miller, S. J. \& Skilleter, G. A. (2006). Temporal variation in habitat use by nekton in a subtropical estuarine system. J. Exp. Mar. Biol. Ecol., 337(1), 82-95. https:// doi.org/10.1016/j.jembe.2006.06.010

Miller, R., Minckley, W. \& Norris, S. (2009). Peces dulceacuícolas de México. México: Comisión Nacional para el Conocimiento y Uso de la Biodiversidad (CONABIO).

Nelson, J. S., Grande, T. C. \& Wilson, M. V. H. (2016). Fishes of the world. Fifth Edition. EE. UU.: Willey \& Sons. https://doi.org/10.1002/9781119174844 
Pérez-Ruzafa, A., Monpéan, C. \& Marcos, C. (2007). Hydrographic, geomorphologic and fish assemblage relationship in coastal lagoons. Hydrobiologia, 577, 107-125. https://doi.org/10.1007/ s10750-006-0421-8

Pielou, E. C. (1966). The measurement of diversity in different types of biological collections. J. Theor. Biol., 13, 131-144. https://doi. org/10.1016/0022-5193(66)90013-0

PNUD (Programa de las Naciones Unidas para el Desarrollo). (2017). Plan de monitoreo y control del pez diablo (Pterygoplichthys spp.) en la RBLT en la Reserva de la Biósfera los Tuxtlas. Aumentar las capacidades nacionales para el manejo de las especies exóticas invasoras (EEI) a través de la implementación de la estrategia nacional de EEI. México: FESAC-Veracruz, PNUD.

Robertson, D. R., Peña, E. A., Posada, J. M. \& Claro, R. (2019). Peces costeros del Gran Caribe: sistema de información en línea. Versión 2. Instituto Smithsonian de Investigaciones Tropicales, Balboa, República de Panamá. https://biogeodb.stri.si.edu/ caribbean/es/thefishes/species/2744

Romero-Berny, E. I., Velázquez-Velázquez, E., Schmitter-Soto, J. J. \& Salgado-Ugarte, I. H. (2020). The influence of habitat on the spatio-temporal dynamics of fish assemblages in a lagoon-estuarine system of the Mexican Pacific. Lat. Amer. J. Aquat. Res., 48(1), 23-37. https://doi.org/10.3856/ vol48-issue1-fulltext-2349

Sánchez-Gil, P., Day, J. W. \& Yáñez-Arancibia, A. (2019). Eco-diversidad: Enfoque ecosistémico costero marino. En E. Rivera-Arriaga, P. Sánchez-Gil \& J. Gutiérrez (Eds.), Tópicos de agenda para la sostenibilidad de costas y mares mexicanos (pp. 1-22). México: UAC-EPOMEX-RED RICOMAR.
Shannon, E. C. \& Wiener, W. (1963). The mathematical theory of communication. EE. UU.: University of Illinois.

Sheridan, P. \& Minello, T. J. (2003). Nekton use of different habitat types seagrasses beds of lower Laguna Madre, Texas. Bull. Mar. Scie., 72, 37-61.

Snedden, G. A., Cable, J. E. \& Kjerfve, B. (2013). Estuarine geomorphology and coastal hydrology. In J. W. Day, B. C. Crump, W. M. Kemp \& A. Yáñez-Arancibia (Eds.), Estuarine ecology (pp. 1938). EE. UU.: Willey \& Sons. https:// doi.org/10.1002/9781118412787.ch2

Solano-Palacios, E. \& Frutos-Cortés, M. (2013). Efectos de la actividad petrolera en el desarrollo regional de Tabasco y Campeche (1970-2008). En R. González-Acolt, A. Sánchez-Almanza, A. Tlacaélel-Ramírez-De León \& F. de J. Salvador-Leal-Medina (Eds.), Desarrollo regional en México. Hacia una agenda para su desarrollo económico y social con sustentabilidad (pp. 239260). México: Universidad Nacional Autónoma de México.

Sosa-Pérez, A., Ramos-Miranda, J., Ayala, L., Flores-Domínguez, D., Villalobos, G. \& Gómez, F. (2012). Cambios de largo plazo en la comunidad de peces y variables fisicoquímicas en la Laguna de Términos, sur del Golfo de México. En A. J. Sánchez, X. Chiappa-Carrara \& R. Brito-Pérez (Eds.), Recursos acuáticos costeros del sureste (pp. 307-330). México: CONCIYTEY-UNAM.

Sparre, P. \& Venema, S. C. (1997). Introduction to tropical fish stock assessment. Part. 1. Manual. Rome: FAO.

Stat Soft, Inc. (2013). STATISTICA. Data Analysis Software System, Version 13. htpp://www.statsoft.com

Ter Braak, C. J. F. \& Verdonschot, P. F. M. (1995). Canonical correspondence analysis and related 
multivariate methods in aquatic ecology. Aquat. Scie., 57, 255-289. https:// doi.org/10.1007/BF00877430

Vaslet, A., Bouchon-Navaro, Y., Charrier, G., Louis, M. \& Bouchon, C. (2010). Spatial patterns of mangrove shoreline fish communities in relation with environmental variables in Caribbean lagoons. Estuar. Coast., 33, 195-210. https://doi. org/10.1007/s12237-009-9225-1

Villanueva, F. S., Vázquez-Botello, A. \& PáezOsuna, F. (1988). Evaluación de algunos metales pesados en organismos del Río Coatzacoalcos y de la Laguna del Ostión, Veracruz, México. Contam. Amb., 4, 19-31.

Yáñez-Arancibia, A., Lara-Domínguez, A. L., Aguirre-León, A., Díaz-Ruiz, S., Amezcua-Linares, F., Flores, D. \& Chavance, P. (1985). Ecology of dominant fish population in tropical estuaries: Environmental factors regulating biological strategies and production.
In A. Yáñez-Arancibia (Ed.), Fish community ecology in estuaries and coastal lagoons: towards an ecosystem integration (pp. 311-366). México: Editorial Universitaria-UNAM.

Yáñez-Arancibia, A. \& Day, J. W. (2010). La zona costera frente al cambio climático: vulnerabilidad de un sistema biocomplejo e implicaciones en el manejo costero. En E. Rivera-Arriaga, I. Azuz-Adeath, L. Alpuche \& G. Villalobos-Zapata (Eds.), Cambio climático en México un enfoque costero-marino (pp. 3-22). México: UAC, Gobierno del Estado de Campeche.

Zar, J. H. (2010). Biostatistical analysis. Fifth Edition. EE. UU.: Prentice-Hall.

Zarate-Hernández, R., Aguirre-León, A., Ortiz-Burgos, S. \& Castillo-Rivera, M. (2007). Ecomorfología de peces estuarinos del Golfo de México. Rev. Contac., 66, 12-20. 
